\title{
Nicolas Sihlé, Rituels bouddhiques de pouvoir et de violence. La figure du tantriste tibétain
}

Turnhout, Brepols Publishers, coll. «Bibliothèque de l'École des Hautes Études, Sciences Religieuses », vol. 152, 2013, 405 p.

\section{Guillaume Rozenberg}

\section{(2) OpenEdition} Journals

Édition électronique

URL : http://journals.openedition.org/assr/27461

DOI : $10.4000 /$ assr.27461

ISSN : $1777-5825$

Éditeur

Éditions de l'EHESS

Édition imprimée

Date de publication : 1 octobre 2015

Pagination : 371

ISBN : 978-2-7132-2515-4

ISSN : 0335-5985

Référence électronique

Guillaume Rozenberg, « Nicolas Sihlé, Rituels bouddhiques de pouvoir et de violence. La figure du tantriste tibétain », Archives de sciences sociales des religions [En ligne], 172 | octobre-décembre, mis en ligne le 21 juin 2016, consulté le 23 septembre 2020. URL : http://journals.openedition.org/assr/27461 ; DOI : https://doi.org/10.4000/assr.27461

Ce document a été généré automatiquement le 23 septembre 2020

(c) Archives de sciences sociales des religions 


\section{Nicolas Sihlé, Rituels bouddhiques de pouvoir et de violence. La figure du tantriste tibétain}

Turnhout, Brepols Publishers, coll. «Bibliothèque de l'École des Hautes Études, Sciences Religieuses », vol. 152, 2013, 405 p.

Guillaume Rozenberg

\section{RÉFÉRENCE}

Nicolas Sihlé, Rituels bouddhiques de pouvoir et de violence. La figure du tantriste tibétain, Turnhout, Brepols Publishers, coll. «Bibliothèque de l'École des Hautes Études, Sciences Religieuses », vol. 152, 2013, 405 p. 
1 À quelles conditions l'exercice de la violence, plus exactement l'acte de mettre à mort des êtres vivants, devient-il légitime dans un contexte socioreligieux où la prohibition d'un tel acte constitue l'élément premier du code éthique auquel est normalement astreint tout fidèle, laïc ou religieux ? Ce problème, Nicolas Sihlé ne s'y est pas heurté dans une situation extrême de conflit ou de guerre entre populations de confession différente. Il s'y est trouvé confronté dans une configuration locale où la violence

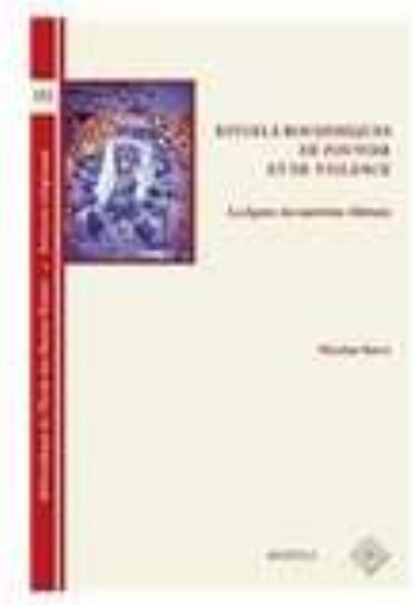
destructrice de vie, de personnes mêmes, malgré l'interdit fondamental qui la vise, représente une composante inhérente à l'ordre socioreligieux. Dans la partie méridionale de la région du Baragaon (nord du Népal) où l'anthropologue a effectué ses enquêtes de terrain dans la deuxième moitié des années 1990, comme au demeurant dans le reste des sociétés appartenant à la sphère culturelle du bouddhisme tibétain, une catégorie majeure de spécialistes religieux se consacre à la pratique de l'exorcisme au sens large, soit des rituels de lutte contre l'infortune, accomplis à titre préventif ou en réaction à une supposée agression surnaturelle. Or cette pratique exorciste inclut, dans ses formes les plus caractéristiques, la spectaculaire mise à mort des agents invisibles, ou "démons ", désignés comme responsables de l'infortune, une mise à mort qui ressortit, d'après l'idéologie bouddhique même dont se réclament les spécialistes concernés, à une grave transgression, un « péché » (selon la traduction, choisie par l'anthropologue, du terme dikpa, d'origine doctrinale) susceptible de conduire son auteur à une réincarnation en enfer. C'est à l'exploration et à l'élucidation de cet apparent paradoxe que s'attache Nicolas Sihlé dans son ouvrage. Mais non à sa résorption. Car le paradoxe en question, montre-t-il au fil de son ethnographie et de ses interprétations, ne saurait être entièrement dissous : il est au fondement de l'identité, particulièrement du pouvoir rituel, des spécialistes de l'exorcisme, dont l'action, aussi répréhensible soit-elle sur un plan éthique, s'avère valorisée parce qu'indispensable à l'existence de la société locale.

Qu'on ne puisse comprendre l'affinité entre l'exercice d'une violence radicale et une certaine catégorie de spécialistes religieux sans considérer la place et le profil singuliers de ces spécialistes dans le paysage socioreligieux local est un des arguments structurants du livre. La première des deux parties examine ainsi la figure du tantriste en tant qu'elle s'inscrit dans une "dualité » avec celle du moine, l'autre spécialiste religieux majeur officiant en contexte tibétain. Les études tibétaines ont souvent préféré l'étude du second au premier, si bien, note l'auteur, que le tantriste demeure encore une figure mal cernée. Par «tantriste» (ngakpa dans la terminologie vernaculaire, littéralement «spécialiste des mantras» et, par extension, des rituels tantriques), il faut entendre un spécialiste religieux que distinguent trois traits. Premièrement, il est maitre de maison et subvient aux besoins de sa famille par une profession mondaine (agriculture, commerce), par contraste avec le moine qui est voué au célibat et a renoncé à toute activité autre que scolastique ou spirituelle. Deuxièmement, le tantriste accède à son rôle par la voie de la filiation patrilinéaire, via 
l'appartenance à un lignage de tantristes, alors que l'ordination monastique ne requiert aucun pedigree préalable. Troisièmement et enfin, quoique sa panoplie rituelle soit étendue, le tantriste est avant tout identifié par sa pratique d'exorcismes violents au service de particuliers ou de la collectivité, dont l'axe de révolution est l'idée d'un pouvoir, d'une force, d'une efficacité mis à disposition des fidèles, tandis que le moine s'illustre principalement, outre dans les rituels funéraires, dans les rituels de lecture de textes canoniques, au centre desquels figure l'idée d'une production de mérites et de bienfaits génériques pour les participants. Quand bien même, reconnaît à plusieurs reprises l'auteur, il convient de ne pas rigidifier à l'excès la dualité entre tantriste et moine - ne serait-ce que parce que les moines sont également susceptibles de réaliser des exorcismes (moins spectaculairement violents cependant) et maittrisent nombre d'autres rituels spécifiques, dits "tantriques", à destination mondaine ou supramondaine - cette dualité n'en organise et n'en structure pas moins, soutient-il, le champ religieux local. Ce constat incite Nicolas Sihlé à nuancer une vision stéréotypique des sociétés bouddhiques, d'ailleurs véhiculée localement, qui conçoit ces sociétés comme régies par l'opposition entre renoncement et vie dans le monde, entre état monastique et état laïc. La figure "ambiguë » du tantriste, qui amalgame inextricablement vie dans le monde et engagement religieux, vient compliquer cette vision. Le tantriste, au contraire du moine, n'est soumis à aucune discipline particulière ; il n'arbore aucun des signes du renoncement. Par son mode d'existence, il ne diffère de facto quasiment pas du laïc ordinaire. Et pourtant, il est, du point de vue indigène, un « homme de religion » (ch'öpa), à l'instar du moine, plutôt qu'un « homme du monde » (jikten).

Dans le monde tibétain, les tantristes d'obédience bouddhique peuvent être issus de lignages isolés, établis au sein de communautés villageoises ordinaires. Au Baragaon méridional toutefois, ensemble de douze villages et de six monastères (trois masculins, trois féminins), ils forment un village à part, Ch'ongkor, comptant une vingtaine de maisons et une centaine d'habitants, principal terrain de Nicolas Sihlé. Les Ch'ongkora appartiennent à un seul et même clan. Ils se réclament d'un ancêtre commun, un maitre puissant, probablement originaire du Tibet central, à qui est attribuée la fondation du village au XviI ${ }^{e}$ siècle. Ils sont à ce point marqués au sceau de l'identité tantriste que tous les hommes du village maîtres de maison (ainsi que leur fils aîné, une fois atteint l'âge de treize ans) jouissent de cette dénomination - d'où la désignation, par l'auteur, de Ch'ongkor comme une "communauté villageoise de tantristes». Toutefois, huit d'entre eux seulement, sur un total de vingt-six, ont accompli l'une au moins des trois retraites qualifiantes pour la fonction d'officiant rituel. Ceux-là sont des «tantristes » et des "religieux» au sens strict, par-delà l'appellation commune et significative de «village de religieux » (ch'öpe lungba) dont se prévalent les Ch'ongkora. Cinq de ces officiants sont en charge des rituels exécutés dans le temple villageois, lhanga («maison des dieux»), pour l'organisation pratique et le financement desquels se mobilisent, à tour de rôle, les maisons du village. C'est dans le temple que se déroule, entre autres, le grand exorcisme annuel Dögyap. Parallèlement à ce versant " collectif » de leur activité rituelle, les officiants se livrent aussi chacun, à la mesure de leur prestige personnel, à des rituels "privés ", au bénéfice de familles de toute la région, avec lesquelles ils entretiennent une relation de patronage régulier. Les tantristes sont fréquemment sollicités par leurs patrons pour effectuer, dans un même temps, le culte de la divinité domestique et le hrinen, rituel prophylactique d'écrasement des démons. 
Une fois circonscrite la figure du tantriste, la seconde partie de l'ouvrage s'intéresse de plus près à son activité d'officiant rituel. Au fondement de cette activité se trouve l'acquisition d'un "savoir-faire " et d'un "pouvoir» rituels. D'une part, le futur tantriste se forme aux divers rituels, à leurs procédures, leurs instruments et leurs danses, en observant et en imitant un officiant aguerri. D'autre part, il accomplit, dans la solitude d'un bâtiment inoccupé du village, les retraites, d'une durée d'une à plusieurs semaines, nécessaires à l'établissement, via la répétition continue de mantras, d'une relation, d'un « rapprochement » (nyenba) avec les divinités tutélaires qu'il aura à mobiliser pour les rituels. Les deuxième et troisième retraites s'accompagnent de rigoureuses pratiques ascétiques (privations alimentaires, obligation de rester assis en tailleur pendant sept jours et sept nuits). L'idée implicite, suggère Nicolas Sihlé, est qu'une « violence faite à son propre corps » détermine l'obtention d'un pouvoir rituel violent. En toute hypothèse, le schème de la retraite se substitue ici à celui de l'initiation (au sens de communication d'un enseignement et donc d'une compétence rituelle par un maître), prévalant dans la diffusion des pratiques tantriques dans le monde tibétain, surtout en contexte monastique. Cette centralité de la retraite dans la transmission d'une spécialité religieuse singularise ainsi un peu plus le tantriste, déjà distingué par le principe de l'accession héréditaire à son rôle (la "lignée de l'os ", disent les intéressés). Font également et notablement partie de la formation du tantriste la connaissance et le maniement d'un certain nombre de textes manuscrits associés aux divinités tutélaires. La lecture des textes pendant les rituels lui permettra de rallier les divinités.

5 L'activité rituelle des tantristes connaît deux modalités, l'une "paisible » (shiwa), l'autre «terrible ", c'est-à-dire violente (dakpo), quoique l'intensité de la violence en question varie selon les cas. Si l'auteur offre un utile panorama de cette activité rituelle, il concentre son attention descriptive et interprétative sur les deux rituels violents emblématiques de la figure du tantriste, impliquant la destruction de démons. La désignation commode de démons embrasse plusieurs catégories d'êtres, peu personnalisés et d'une nature relativement floue, que caractérise une agressivité débridée, source pour les humains d'infortunes multiples, et qui sont conçus comme des ennemis de la doctrine bouddhique. Le hrinen, rituel d'écrasement des démons hri exécuté par un tantriste à la demande d'un de ses patrons à titre de protection générale contre les menaces d'infortune et éventuellement, par inclusion, d'opération sorcellaire contre un humain, voit l'officiant, divinités tutélaires à l'appui, accumuler les moyens violents pour exclure de la maison et exterminer les démons (ainsi que la cible humaine, le cas échéant), représentés par des effigies en pâte. "Dans un tintamarre effrayant, les effigies sont renversées, bombardées de substances toxiques, découpées, enfoncées et scellées dans un crâne. L'ennemi est menacé d'armes diverses, ligoté, empalé, découpé en morceaux, consommé par les divinités et l'officiant luimême ». Puis, " expulsé hors de la maison après un dernier rite de repoussement, [il est] enfin enterré, écrasé sous terre, emprisonné sous de lourdes dalles et sous le feu » (p. 188). Le grand exorcisme Dögyap - reprise à leur compte par les Ch'ongkora d'un rituel qu'ils réalisaient jusqu'aux années 1920 dans un bourg voisin au bénéfice de toute la région - a lieu pour sa part dans le temple du village. S'étendant sur cinq jours, il purifie la communauté locale de l'ensemble des influences néfastes génératrices de désordre et d'infortune. Il est considéré comme le plus puissant des exorcismes. Là encore, des effigies sont l'objet de traitements destructeurs et de mise à mort, une expulsion du village. 
6 L'importance des textes dans ces rituels entrâne Nicolas Sihlé dans des réflexions éclairantes sur leurs origines, leur composition, leur degré d'(in)intelligibilité et leurs usages dans le cadre rituel - contribution à ce qu'il qualifie d'«anthropologie de l'écrit ». À voir comment les rituels sont souvent «bricolés » à partir de textes et de séquences manifestement extraits de cycles distincts, l'anthropologue aurait tort, nous avertit l'auteur, de chercher à tout prix une cohérence parfaite. L'opacité du phénomène rituel semble comme exacerbée dans ce contexte, où l'intense circulation des textes et des pratiques à l'échelle de l'aire culturelle tibétaine a alimenté, dans un milieu relativement peu institutionnalisé comme le tantrisme de Ch'ongkor, des "synthèses " locales instables dont il paraît sans doute vain de vouloir mettre en évidence une logique et une structure générales. Des distinctions telles que religion des élites versus religion populaire, religion universelle versus religion locale, ou bien encore grande tradition versus petite tradition ne rendent en tout cas pas justice, d'après Nicolas Sihlé, à une telle dynamique, qui fait des rituels ch'ongkora et de leurs officiants des "points d'articulation" entre le monde englobant du bouddhisme tibétain et le contexte socioreligieux du Baragaon méridional.

7 Ayant envisagé le rapport entre tantristes et violence rituelle sous tous ses angles, Nicolas Sihlé revient alors au point de départ de son étude, le problème de l'exercice récurrent et reconnu d'une violence destructrice de vie dans un contexte socioreligieux qui proscrit théoriquement une telle violence. Les tantristes, rappelle-t-il d'abord, ne sauraient être réduits à cette violence. Leur qualité de spécialiste religieux inclut également des traits non problématiques tels que la dispensation de bienfaits et des références symboliques à l'idéal bouddhique, notamment monastique. Néanmoins, les rituels les plus violents constituent leur attribut distinctif et l'imaginaire local les associe significativement à une pratique aussi redoutable et blâmable que la sorcellerie. L'auteur fait alors valoir la pluralité locale des « évaluations morales » relatives à cette violence. D'aucuns, assez rares, et extérieurs à Ch'ongkor, jugent l'exorcisme dénué de tout péché (dikpa) : la mise à mort des démons serait au contraire une action vertueuse permettant leur délivrance par une renaissance dans un état meilleur, dans une terre pure de bouddha. La majorité des gens du Baragaon cependant, dont les Ch'ongkora, peuvent exprimer un malaise vis-à-vis d'un rituel comme le hrinen, à leurs yeux foncièrement empreint de péché (dikpa), tout en ne donnant pas forcément au terme le même contenu. Un tel malaise semble avoir été, sinon engendré, du moins nourri par le discours d'un moine tibétain influent localement qui, dans les années 1960, condamna fermement le hrinen, avec pour conséquence un abandon par certaines maisons du recours à ce rituel. En même temps, cette idée de péché concernant le hrinen demeure quelque peu lointaine et marginale dans les préoccupations de nombre de bouddhistes. Elle ne parvient pas à ébranler les tantristes et leurs patrons actuels au point qu'ils décident d'en finir avec le hrinen. Car, de leur point de vue, « dans une certaine mesure, tuer des démons est une violence légitime face à la violence du monde» (p. 243), légitime parce que son enjeu est tout simplement la vie. Et le tantriste s'avère essentiellement apte à déployer cette violence légitime du fait même de l'ambiguïté de sa condition. Pour Nicolas Sihlé, en effet, l'identité complexe du tantriste, entre "homme du monde» et "homme de religion», recèle une clé explicative de sa spécialisation relativement paradoxale. Familier du péché par son statut de maître de maison en même temps que détenteur d'un pouvoir rituel singulier par sa formation, le tantriste serait le mieux placé pour assumer la part de violence rituelle nécessaire à la défense du bouddhisme et des bouddhistes. Il autoriserait par là le moine à préserver sa 
« pureté » et donc son « éminence morale », qui sous-tendent sa capacité à produire des mérites.

8 L'ouvrage s'achève par une double perspective comparative. L'auteur fait dans un premier temps ressortir la spécificité de la figure du tantriste tibétain vis-à-vis de ses homologues, pratiquant l'exorcisme dans d'autres contextes bouddhiques, non tibétains. Nulle part ne s'observe dans les rituels une violence destructrice de vie analogue à celle du tantriste. Mais l'anthropologue propose, dans un deuxième temps, de dépasser cette spécificité pour formuler, à partir du cas tibétain et d'un rapprochement avec des configurations indiennes cette fois-ci, une manière possible de conceptualiser le champ religieux dans nombre de sociétés de par le monde où, semblablement à la dualité entre moine et tantriste, existerait une dualité entre un pôle de la "pureté ", incarné par un type de spécialiste au « statut social prééminent », et un pôle du " pouvoir rituel fort, voire violent ", incarné par un autre type de spécialiste, à "l'orientation religieuse éthiquement ambivalente, marquée par l'impureté et la transgression » (p. 272). C'est là, bien entendu, l'aspect le plus spéculatif d'un ouvrage très maîtrisé, jouissant qui plus est d'un soin remarquable apporté à son édition (avec plusieurs annexes, un glossaire complet, un impressionnant index de trente pages, et un riche cahier d'illustrations). Si les notions de "pureté " et de "pouvoir rituel» souffrent peut-être d'une trop grande polysémie pour être pleinement efficaces sur le plan d'une caractérisation comparative, gageons que la proposition de Nicolas Sihlé, arc-boutée sur un travail de recherche substantiel, représentera une référence stimulante pour les réflexions futures sur le fait et le champ religieux. 